\title{
VALIDATION OF THE SENTINEL-3 OCEAN AND LAND COLOUR INSTRUMENT (OLCI) TERRESTRIAL CHLOROPHYLL INDEX (OTCI): SYNERGETIC EXPLOITATION OF THE SENTINEL-2 MISSIONS
}

\author{
Luke A. Brown ${ }^{1 *}$, Jadunandan Dash ${ }^{1}$, Antonio L. Lidón ${ }^{2}$, Ernesto Lopez-Baeza ${ }^{3}$ and Steffen Dransfeld ${ }^{4}$ \\ ${ }^{1}$ Geography and Environment, University of Southampton, *1.a.brown@ soton.ac.uk \\ ${ }^{2}$ Department of Hydraulic Engineering and Environment, Universitat Politècnica de València \\ ${ }^{3}$ Department of Earth Physics and Thermodynamics, University of Valencia \\ ${ }^{4}$ European Space Research Institute, European Space Agency
}

\begin{abstract}
Continuity to the Medium Resolution Imaging Spectrometer (MERIS) Terrestrial Chlorophyll Index (MTCI) will be provided by the Sentinel-3 Ocean and Land Colour Instrument (OLCI), and to ensure its utility in a wide range of operational applications, validation efforts are required. In the past, these activities have been constrained by the need for costly airborne hyperspectral data acquisition, but the Sentinel-2 Multispectral Instrument (MSI) now offers a promising alternative. In this paper, we explore the synergetic use of Sentinel-2 MSI data for validation of the Sentinel-3 OLCI Terrestrial Chlorophyll Index (OTCI) over the Valencia Anchor Station, a large agricultural site in the Valencian Community, Spain. High retrieval accuracy $\left(R M S E=0.20 \mathrm{~g} \mathrm{~m}^{-2}\right)$ was obtained by applying machine learning techniques to Sentinel-2 MSI data, highlighting the valuable information it can provide when used in synergy with Sentinel-3 OLCI data for land product validation.
\end{abstract}

Index Terms - Vegetation biophysical variables, canopy chlorophyll content, Sentinel-2, Sentinel-3, validation

\section{INTRODUCTION}

Making use of the red-edge bands of the Medium Resolution Imaging Spectrometer (MERIS), the MERIS Terrestrial Chlorophyll Index (MTCI) provided the first global surrogate of canopy chlorophyll content (CCC) at a spatial resolution of $300 \mathrm{~m} \mathrm{[1].} \mathrm{Continuity} \mathrm{to} \mathrm{its} 10$ year archive will provided by the Sentinel-3 Ocean and Land Colour Instrument (OLCI) in the form of the OLCI Terrestrial Chlorophyll Index (OTCI), but to ensure its utility in a wide range of operational applications, validation efforts are required to quantify its accuracy and uncertainty.

The moderate spatial resolution of satellite-derived CCC products and the heterogeneity of the terrestrial landscape make validation particularly challenging, as field measurements are typically point-based. The 'two-stage' or 'bottom-up' approach, in which high spatial resolution imagery is used to bridge this scale gap, was developed to address these challenges [2], [3]. However, prior to the launch of the Sentinel-2 missions, its application to satellitederived CCC products was constrained by a lack of freely available high spatial resolution imagery incorporating appropriate spectral bands. Validation efforts necessitated costly airborne hyperspectral data acquisition, making them a relatively infrequent activity. With a spatial resolution of $20 \mathrm{~m}$ in multiple red-edge bands, the Sentinel-2 Multispectral Instrument (MSI) offers a promising alternative in this respect. As such, in this paper, we explore the synergetic use of Senitnel-2 MSI data for validation of the Sentinel-3 OTCI.

\section{MATERIALS AND METHODS}

\subsection{Field data collection}

Field data collection was carried out between $14^{\text {th }}$ and $18^{\text {th }}$ June 2017 within a $10 \mathrm{~km} \mathrm{x} 10 \mathrm{~km}$ area of the Valencia Anchor Station, a large vineyard dominated agricultural site in the Valencian Community, Spain (39.5707, -1.2882). 26 grapevine elementary sampling units (ESUs) were established over the study site, in which measurements of leaf area index (LAI) and leaf chlorophyll concentration (LCC) were obtained. Each ESU was approximately $40 \mathrm{~m} \mathrm{x}$ $40 \mathrm{~m}$ in size, enabling positional uncertainties in the $20 \mathrm{~m}$ MSI data to be accounted for [4]. Using a handheld global positioning system (GPS) device (Garmin eTrex 10), the location of each ESU was determined with an error of $<10$ $\mathrm{m}$.

Within each ESU, 20 measurements of LAI were obtained using digital hemispherical photography (Nikon Coolpix 4500 with FC-E8 fisheye lens), whilst 10 measurements of LCC (each comprised of 18 replicates) were obtained using an optical chlorophyll meter (Konica Minolta SPAD-502). These measurements were conducted over 5 transects positioned diagonally with respect to 
vineyard orientation, enabling the row structure of the canopy to be characterised. DHP data were binarised and processed to yield estimates of LAI according to [5]. Images were split into 6 zenith rings of $10^{\circ}$ and each zenith ring into a further 36 azimuth cells of $10^{\circ}$, whilst zenith angles of $>60^{\circ}$ were discarded due to the likelihood of mixed pixels [6]. The method proposed by [7] was adopted to account for the effects of foliage clumping. In terms of LCC, the relative values provided by the optical chlorophyll meter were converted to physical units using the calibration function reported for grapevine by [8]. CCC was then derived as the product of LAI and LCC.

\subsection{Generation of a high spatial resolution $\mathrm{CCC}$ reference map from Sentinel-2 MSI data}

A machine learning approach was used to generate a high spatial resolution CCC reference map from L2A Sentinel-2A MSI data acquired on $15^{\text {th }}$ June 2017. CCC was retrieved using an artificial neural network (ANN), trained using the coupled Leaf Optical Properties Spectra (PROSPECT) and Scattering by Arbitrarily Inclined Leaves (SAIL) radiative transfer models (RTMs), with additions to account for row structured canopies [9]-[13]. 50,000 simulations were carried out by randomly drawing input parameters from within predefined value ranges (Table 1). The soil background was selected randomly from 10 possible spectra reflecting the sandy loam soils of the study site [14]. Simulated spectra were resampled according to the MSI spectral response functions [15], and were contaminated with wavelength dependent and independent Gaussian noise (0.01 additive, 2\% multiplicative) to better account for instrument uncertainties.

Table 1: Range of values from which RTM input parameters were randomly drawn.

\begin{tabular}{lll}
\hline Parameter & Values & Reference \\
\hline Structure $(\mathrm{N})$ & 1.62 & {$[12],[13]$} \\
Chlorophyll $\mathrm{a}+\mathrm{b}\left(\mu \mathrm{g} \mathrm{cm}^{-2}\right)$ & 27.4 to 43.7 & This study \\
Water thickness $(\mathrm{cm})$ & 0.025 & {$[12],[13]$} \\
Dry matter $\left(\mathrm{g} \mathrm{cm}^{-2}\right)$ & 0.0035 & {$[12],[13]$} \\
Leaf area index & 0.7 to 3 & This study \\
Average leaf angle $\left(^{\circ}\right)$ & 45 & {$[12],[13]$} \\
Hotspot parameter & 0.083 & {$[12],[13]$} \\
Row height $(\mathrm{m})$ & 1.2 to 1.8 & {$[12],[13]$} \\
Crown diameter $(\mathrm{m})$ & 0.6 to 1.3 & {$[12],[13]$} \\
Visible soil strip $(\mathrm{m})$ & 1.5 to 3 & This study \\
Difference between solar & 0.3 to 125.7 & This study \\
azimuth angle and row & & \\
direction $\left({ }^{\circ}\right)$ & & \\
Observer zenith angle $\left(^{\circ}\right)$ & 5.5 to 6.8 & This study \\
Solar zenith angle $\left({ }^{\circ}\right)$ & 21.3 to 21.5 & This study \\
Relative azimuth angle $\left(^{\circ}\right)$ & 18.9 to 44.1 & This study \\
Fraction of diffuse radiation & 0.15 & This study \\
\hline
\end{tabular}

The ANN was comprised of a single hidden layer with 5 tangent sigmoid neurons. Inputs consisted of the bottom-of-atmosphere reflectance values in MSI bands 3, 4, $5,6,7,8 \mathrm{a}, 11$ and 12 , in addition to the cosine of the observer zenith angle, solar zenith angle and relative azimuth angle. 50\% of simulations were used for training, and the remaining $50 \%$ were used for validation and testing. Once trained, the ANN was applied to the MSI scene (Figure 1). The resulting CCC retrievals were validated against the field measurements, and retrieval accuracy was assessed in terms of the root mean square error (RMSE).

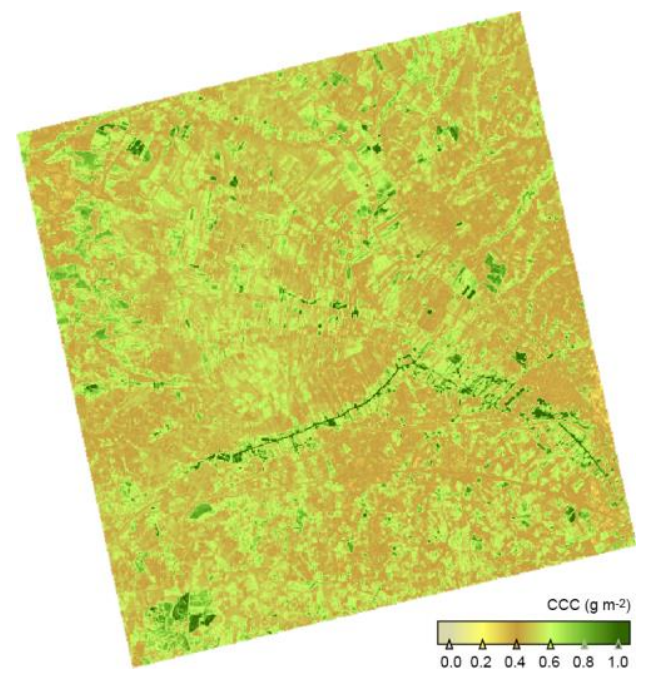

Figure 1: 20 m MSI-derived CCC reference map.

\subsection{Validation of the Sentinel-3 OTCI}

L2 Sentinel-3A OLCI data were acquired on $18^{\text {th }}$ June 2017. Estimates of CCC were derived from OTCI values using empirical relationships established in previous validation efforts for the MTCI [16], [17]. Due to positional uncertainties in the L2 OLCI data, quantitative comparison could not be reliably conducted at its native spatial resolution. Following [16], both the $20 \mathrm{~m}$ MSI-derived CCC reference map and $300 \mathrm{~m} \mathrm{L2}$ OLCI scene were aggregated to a common spatial resolution of $1 \mathrm{~km}$. These aggregated datasets were then collocated, and performance was again assessed in terms of the RMSE.

\section{RESULTS AND DISCUSSION}

\subsection{Accuracy of the $20 \mathrm{~m}$ MSI-derived CCC reference map}

The $20 \mathrm{~m}$ MSI-derived CCC reference map demonstrated good spatial consistency with observed patterns of vegetation cover over the study site (Figure 1). A strong linear relationship between CCC retrievals and field measurements was observed $(r=0.66)$, although in most cases the ANN slightly overestimated CCC (Figure 2). 
Nevertheless, a high retrieval accuracy was obtained (RMSE $=0.20 \mathrm{~g} \mathrm{~m}^{-2}$ ). Comparable retrieval accuracies were achieved by [18], who applied RTM inversion techniques to $5 \mathrm{~m}$ RapidEye data $\left(\mathrm{RMSE}=0.39\right.$ to $0.43 \mathrm{~g} \mathrm{~m}^{-2}$ ).

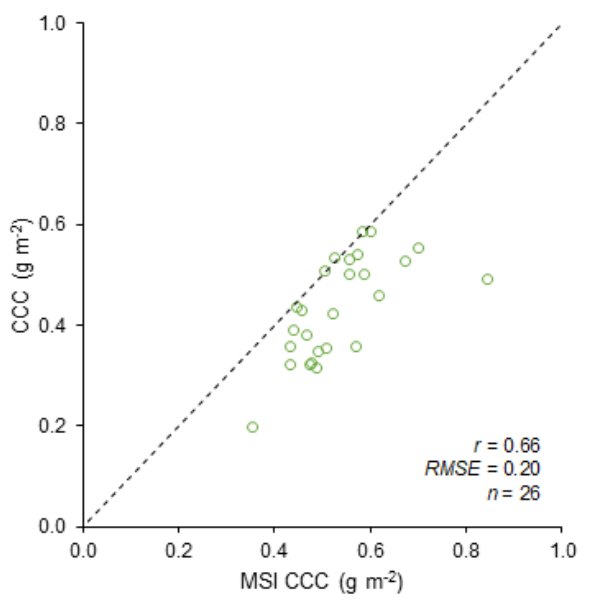

Figure 2: Relationship between field measurements and MSI-derived CCC retrievals. Dashed line represents a 1:1 relationship.

\subsection{Performance of the OTCI}

Overall, reasonable spatial consistency was observed between the aggregated MSI-derived CCC reference map and OTCI-derived estimates of CCC, which resolved major spatial structures over the study site (Figure 3). Although only a moderate linear relationship between the OTCI and CCC was observed $(r=0.37)$, when estimates of CCC were derived from the OTCI using previously established empirical relationships, good performance was demonstrated (Figure 4). Using the empirical relationship reported by [16], an RMSE of $0.24 \mathrm{~g} \mathrm{~m}^{-2}$ was obtained, whilst an improved RMSE of $0.20 \mathrm{~g} \mathrm{~m}^{-2}$ was achieved using that of [17]. The moderate nature of the relationship between the OTCI and CCC observed in this study is primarily a result of the limited range of CCC values experienced over the study site. This is particularly apparent when compared to previous validation efforts, which have incorporated CCC values of up to $3.5 \mathrm{~g} \mathrm{~m}^{-2}$ [16], [18].

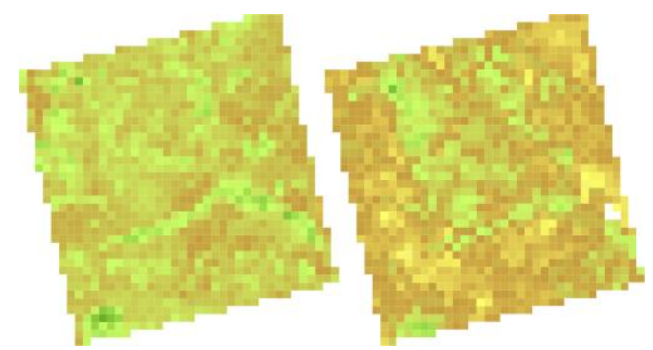

Figure 3: $300 \mathrm{~m}$ aggregated MSI-derived CCC reference map (left) and OTCI-derived CCC (right). See Figure 1 for interpretation of color scale.

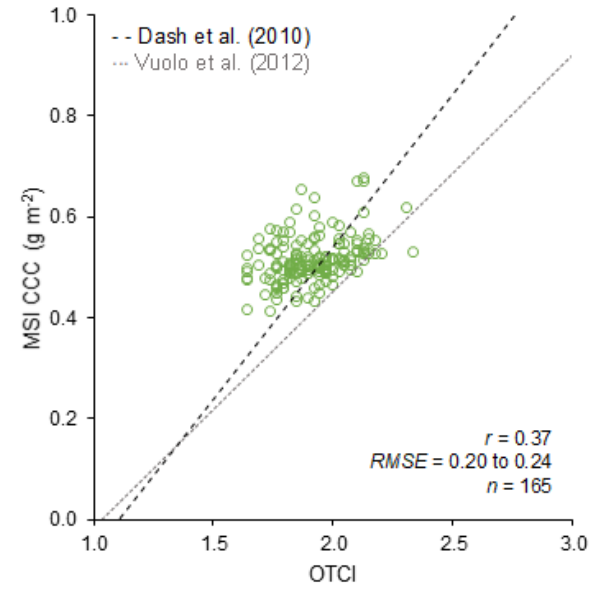

Figure 4: Relationship between MSI-derived CCC retrievals and the OTCI, aggregated at $1 \mathrm{~km}$. Dashed and dotted lines represent the empirical relationships reported in [16] and

[17].

\subsection{Synergetic potential of the Sentinel-2 missions for validation of Sentinel-3 OLCI land products}

The results of this study indicate that Sentinel-2 MSI data are well suited to the retrieval of vegetation biophysical variables such as CCC, and thus provide valuable information that can be used in synergy with Sentinel-3 OLCI data for the purposes of land product validation. When compared to infrequent airborne hyperspectral data acquisition, the Sentinel-2 missions represent a major advance towards the routine validation of satellite-derived $\mathrm{CCC}$ products. Although not directly investigated in this study, the red-edge bands provided by MSI open up opportunities for deriving spectral vegetation indices similar to the OTCI at a higher spatial resolution than previously possible [19]. With appropriate consideration of positional uncertainties and differences in spectral response [20], this capability could be further exploited for inter-mission comparison.

\section{CONCLUSIONS}

The synergetic use of Senitnel-2 MSI data for validation of the Sentinel-3 OTCI was explored over a large agricultural site in the Valencian Community, Spain. A framework for validating moderate spatial resolution CCC products using Sentinel-2 MSI data was established, and its suitability for upscaling field measurements was evaluated. Importantly, the high retrieval accuracy obtained by applying machine learning techniques to Sentinel-2 MSI data highlights the valuable information it can provide on vegetation biophysical variables. In the context of Sentinel-3 OLCI land product validation, the Sentinel-2 missions are a key facilitator of operational validation activities. Further work 
is required to evaluate the proposed framework over additional sites covering a wide range of vegetation types.

\section{ACKNOWLEDGEMENTS}

The authors thank the European Space Agency and European Commission for making the Sentinel products and associated tools freely available through the Copernicus programme. The authors are also grateful to the Sentinel-3 Mission Performance Centre, and to Francesco D'Adamo, Andrew MacLachlan, Harry Morris, Erika Albero, Pilar Campins, Domingo Catalan, Amparo Coll, Amparo Gilabert, Beatriz Martinez, Sergio Sanchez, Aida Vicente, Inmaculada Bautista, Cristina Lull, Jennifer Adams, Nadine Gobron, Christian Lanconelli, Olivier Morgan and Domingo Iglesias for their assistance in field campaign planning and execution.

\section{REFERENCES}

[1] J. Dash and P. J. Curran, "The MERIS terrestrial chlorophyll index," Int. J. Remote Sens., vol. 25, no. 23, pp. 5403-5413, Dec. 2004.

[2] J. T. Morisette et al., "Validation of global moderate-resolution LAI products: a framework proposed within the CEOS land product validation subgroup," IEEE Trans. Geosci. Remote Sens., vol. 44, no. 7, pp. 1804-1817, Jul. 2006.

[3] R. Fernandes et al., "Global Leaf Area Index Product Validation Good Practices," in Best Practice for Satellite-Derived Land Product Validation, 2.0., R. Fernandes, S. Plummer, and J. Nightingale, Eds. Land Product Validation Subgroup (Committee on Earth Observation Satellites Working Group on Calibration and Validation), 2014.

[4] F. Gascon et al., "Copernicus Sentinel-2 Calibration and Products Validation Status," Frascati, Italy, 2016.

[5] J. Miller, "A formula for average foliage density," Aust. J. Bot., vol. 15, no. 1, pp. 141-144, Apr. 1967.

[6] M. Weiss and F. Baret, CAN-EYE V6.313 User Manual. Avignon, France: Institut National de la Recherche Agronomique, 2010.

[7] A. R. G. Lang and X. Yueqin, "Estimation of leaf area index from transmission of direct sunlight in discontinuous canopies," Agric. For. Meteorol., vol. 37, no. 3, pp. 229-243, Aug. 1986.

[8] M. R. Steele, A. A. Gitelson, and D. C. Rundquist, "A Comparison of Two Techniques for Nondestructive Measurement of Chlorophyll Content in Grapevine Leaves," Agron. J., vol. 100, no. 3, p. 779, 2008.

[9] S. Jacquemoud and F. Baret, "PROSPECT: A model of leaf optical properties spectra," Remote Sens.
Environ., vol. 34, no. 2, pp. 75-91, Nov. 1990.

[10] W. Verhoef, L. Jia, Q. Xiao, and Z. Su, "Unified Optical-Thermal Four-Stream Radiative Transfer Theory for Homogeneous Vegetation Canopies," IEEE Trans. Geosci. Remote Sens., vol. 45, no. 6, pp. 1808-1822, Jun. 2007.

[11] S. Jacquemoud et al., "PROSPECT+SAIL models: A review of use for vegetation characterization," Remote Sens. Environ., vol. 113, no. SUPPL. 1, pp. S56-S66, Sep. 2009.

[12] P. J. Zarco-Tejada et al., "Assessing vineyard condition with hyperspectral indices: Leaf and canopy reflectance simulation in a row-structured discontinuous canopy," Remote Sens. Environ., vol. 99, no. 3, pp. 271-287, Nov. 2005.

[13] F. Meggio, P. J. Zarco-Tejada, J. R. Miller, P. Martín, M. R. González, and A. Berjón, "Row orientation and viewing geometry effects on rowstructured vine crops for chlorophyll content estimation," Can. J. Remote Sens., vol. 34, no. 3, pp. 220-234, 2008.

[14] A. M. Baldridge, S. J. Hook, C. I. Grove, and G. Rivera, "The ASTER spectral library version 2.0," Remote Sens. Environ., vol. 113, no. 4, pp. 711715, Apr. 2009.

[15] ESA, "Sentinel-2 Spectral Response Functions (S2SRF)," 2017. [Online]. Available: https://earth.esa.int/documents/247904/685211/Senti nel-2+MSI+Spectral+Responses/. [Accessed: 16May-2017].

[16] F. Vuolo, J. Dash, P. J. Curran, D. Lajas, and E. Kwiatkowska, "Methodologies and Uncertainties in the Use of the Terrestrial Chlorophyll Index for the Sentinel-3 Mission," Remote Sens., vol. 4, no. 12, pp. 1112-1133, Apr. 2012.

[17] J. Dash, P. J. Curran, M. J. Tallis, G. M. Llewellyn, G. Taylor, and P. Snoeij, "Validating the MERIS Terrestrial Chlorophyll Index (MTCI) with ground chlorophyll content data at MERIS spatial resolution," Int. J. Remote Sens., vol. 31, no. 20, pp. 5513-5532, Oct. 2010.

[18] F. Vuolo, C. Atzberger, K. Richter, and J. Dash, "Retrieval of Biophysical Vegetation Products From Rapideye Imagery," in Proceedings of the ISPRS TC VII Symposium, 2010, pp. 281-286.

[19] W. J. Frampton, J. Dash, G. Watmough, and E. J. Milton, "Evaluating the capabilities of Sentinel-2 for quantitative estimation of biophysical variables in vegetation," ISPRS J. Photogramm. Remote Sens., vol. 82, pp. 83-92, Aug. 2013.

[20] G. Chander et al., "Applications of Spectral Band Adjustment Factors (SBAF) for Cross-Calibration," IEEE Trans. Geosci. Remote Sens., vol. 51, no. 3, pp. 1267-1281, Mar. 2013. 